\title{
Potential pathogenesis of multiple organ injury in COVID-19
}

\author{
${ }^{* 1}$ Ling Guo, ${ }^{1}$ Kui-peng,Yu,,${ }^{1}$ Deng-ren Li, ${ }^{1}$ Huimin Yang, ${ }^{1}$ Lei Liu, ${ }^{1}$ Jia-hui Fan, ${ }^{1}$ Nan, Sun, ${ }^{* *}$ Xiang-
} dong Yang

${ }^{1}$ Department of Nephrology, Qilu Hospital of Shandong University, No 107 Wenhua West

Road, Ji'nan, 250012

*The first author

** Corresponding author: Xiang-Dong Yang, Email: yxd683@163.com.

${ }^{1}$ Department of Nephrology, Qilu Hospital of Shandong University, Jinan, 250021 Shandon g, China.

**Department of Nephrology, Qilu Hospital of Shandong University, No 107 Wenhua West Road, Ji'nan, 250012, China.

Fund: This work was supported by grants from the National Natural Science Foundation (81670660), Department of Science \& Technology of Shandong province (2019GSF108087) of Shandong Province of China.

\begin{abstract}
Novel coronavirus (COVID-19) can lead to multiple organ injuries such as acute respiratory distress syndrome(ARDS), acute renal injury(AKI) and so on. ACE2 is an important part of the renin-angiotensin system (RAS) and a key protein needed for COVID-19 to invade cells. First of all, we searched the HPA, GTEx and FANTOM5 Databases and found that the expression of ACE2 in kidney tissue was significantly higher than that in lung tissue. Then, by searching the Nephroseq Database, it is further verified that ACE2 is highly expressed in renal tissue and plays a protective role in renal tissue. However, current studies have found that the incidence of AKI caused by COVID-19 is much lower than that of ARDS. Because of this, we further searched the proteins interacting with ACE2 protein through the STING Database and analyzed the expression of tissue protein mRNA in the HPA Database. It was noted that AGTR2 mRNA was highly expressed in lung tissue, but low in kidney tissue, and hard tissue specificity in lung tissue. Through further research, it is found that AGTR2 plays a major role in the development of pulmonary fibrosis. Therefore, AGTR2 may be a key protein in COVID-19 pneumonia, and AGTR2 may be a potential new therapeutic target for the treatment of COVID-19 patients.
\end{abstract}

Keyword:AGTR2；ACE2；COVID-19 


\section{Introduction}

In December 2019, a cluster of acute respiratory illness was named by WH O COVID-19, occurred in Wuhan, Hubei Province, China ${ }^{[1]}$, which raised inten se attention not only within China but internationally ${ }^{[2]}$. However, the pathogen esis of COVID-19 is complex that has not been fully elucidated ${ }^{[3]}$. There is no specific antiviral treatment or vaccine, leading some patients died of multiple organ failure ${ }^{[4-7]}$.

ACE2 is an angiotensin-converting enzyme (ACE) isozyme, which negatively regulates the activated renin-angiotensin system by degrading Ang II into Ang $1-7^{[8]}$, which plays an important protective effect in kidney and cardiovascular system, and lung ${ }^{[9]}$. Studies have revealed that COVID-19 enters host cells thro ugh ACE2 protein ${ }^{[10,11]}$.ACE2 protein becomes the receptor of virus infection ${ }^{[12,}$ 13]. Therefore, cells with high expression of ACE2 may become damaged targe $\mathrm{t}$ cells and are susceptible to COVID-19 infection ${ }^{[14]}$.

Our team found that ACE2 protein was abundantly expressed in renal tubule $\mathrm{s}$, but not in lung tissue. The expression of ACE2 in kidney tissue was signifi cantly higher than that in lung tissue. However, lung tissue has become the pri ncipal infectious organ of COVID-19, and the complication of (ARDS) in acut e respiratory distress syndrome is much higher than that of (AKI) in acute ren al injury. We have conducted a study on whether other mechanisms mediate th e entry of COVID-19 into cells and lead to lung tissue injury.

\section{Methods}

\section{Expression of ACE2 in tissues.}

The gene expression profile of ACE2 in normal tissues was obtained from The Human PROTEIN Atlas (HPA Database: https://www.proteinatlas.org/). ACE2 mRNA in lung tissue and kidney tissue were compared and discussed. Further verification is performed through GTEx, FANTOM5 and Nephroseq Databases. At the same time, the expression of ACE2 protein in the lung and kidney was further verified by the HPA Database and immunohistochemical.

\section{The role of $\mathrm{ACE} 2$ in kidney tissue.}

The expression of ACE2 in the kidney was further verified from the Nephrose q Database (https://www.nephroseq.org/resource/main.html). Using the Nephroseq Database, the expression of ACE2 mRNA and serum creatinine, estimated glom erular filtration rate (eGFR), urinary protein and mean blood pressure were ana lyzed to determine the role of ACE2 in renal tissue.

Clinical data of AKI and ARDS brought about by COVID-19. Web sites of "Pubmed Database" and "BioRxiv" and "medRxiv" were reprinted online, the keywords were "COVID-19", "nCoV" and "novel coronavirus". Clinical informa tion was collected and the data of ARDS and AKI caused by COVID-19 were compared.

ACE2 protein relationship network and the role of AGTR2 in lung tissue. Using the STRING Database (https://string-db.org), taking people as the researc $\mathrm{h}$ object. The visual association network of ACE2 and its related proteins was constructed. According to the string protein relationship network of ACE2, it w 
as found that 11 proteins were closely related to the function of ACE2 protein s. In further searching the HPA Database, we found the tissue-specific expressi on of 11 protein genes in different organs, which were used for further researc $\mathrm{h}$ and analysis, and found that AGTR2 mRNA was specifically expressed in lu ng tissue. For this reason, we further searched the role and mechanism of AG TR2 in lung tissue by the Pubmed to clarify the importance of AGTR2 in lun g tissue.

\section{Results}

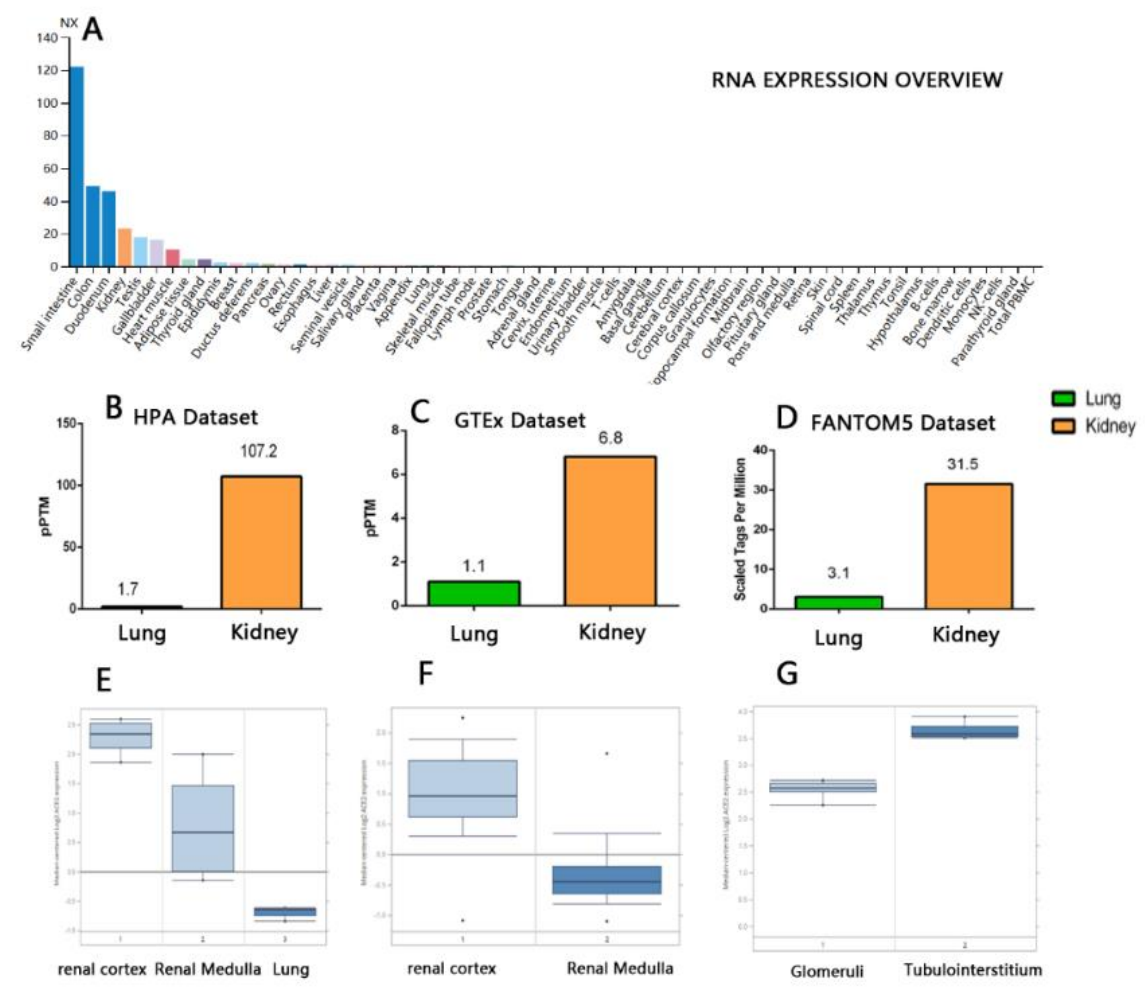

Fig.1 A: Expression of ACE2 mRNA in tissues. (HPA Database). B-D: The exp ression of ACE2 mRNA in lung and kidney. (HPA Database, GTEx Database, F ANTOM5 Database). E: The expression of ACE2 mRNA in the renal cortex (n $=4)$, renal medulla $(n=4)$ and lung tissue $(n=3)(P=4.81 e-4) . F$ : The expression of ACE2 mRNA in the renal cortex (nasty 72) and renal medulla (nasty 61) $(\mathrm{P}=8$. 49e-24). G: The expression of ACE2 mRNA in glomeruli and tubules ( $\mathrm{P}=4.08 \mathrm{e}-$ 7). (E-F:Data source Nephroseq Database)

The expression of ACE2 mRNA in kidney tissue was higher than that in I ung tissue, and mainly in renal tubules.

The expression of ACE2 mRNA is the highest in the digestive system (small $\mathrm{i}$ ntestine, colon, duodenum), which also accounts for this cause of gastrointestin al symptoms such as diarrhea in patients with COVID-19 ${ }^{[15,16]}$. Secondly, ACE 2 mRNA is mainly highly expressed in the kidney, testis, gallbladder and other organs. (fig. 1A) of the expression of ACE2 mRNA in lung tissue is low. In this regard, we get to make a systematic comparison and analysis of renal tiss ue and lung tissue. The expression of ACE2 mRNA was 1.7 pPTM in lung tis 
sue and 107.2pPTM in kidney tissue. (figure 1B-HPA Dataset), further proved $t$ hat the expression of ACE2 mRNA in kidney tissue was greater than in lung $t$ issue through the GTEx Database (figure 1C), FANTOM5 Database (figure 1D) and Nephroseq Database (figure 1E).Through the Nephroseq Database, the me dian expression of ACE2 mRNA was 0.956 in the renal cortex $(n=72)$ and -0 . 450 in the medulla( $n=61)$. The median expression of ACE2 mRNA was 2.570 in glomeruli $(n=6)$ and 3.576 in renal tubules $(n=6)(p<0.01)$. It proves that $A C E$ 2 was mainly expressed in proximal cortical tubules.
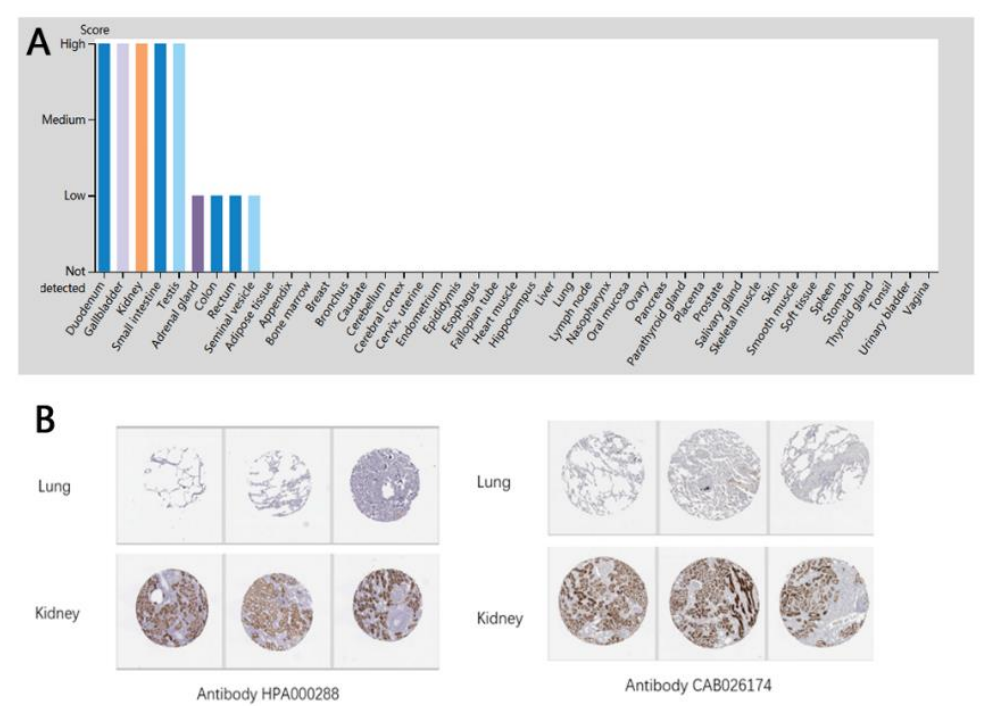

Fig.2 The expression of ACE2 protein in tissue. A: The expression of ACE2 protein in normal lung and kidney tissues. B: Immunohistochemical of AntibodyHPA0002 and AntibodyCAB026174 in lung and kidney tissues (HPA Database)

The expression of ACE2 protein in kidney tissue was higher than that in 1 ung tissue.

The expression of ACE2 protein was the highest in the gallbladder, small intes tine, kidney and testis, attended by the adrenal gland, colon, rectum, and semin al vesicle. However, there is not any expression of ACE2 protein in lung tissu e and other tissues (figure 2A). The expression of ACE2 protein is basically c onsistent with that of ACE2mRNA in tissues. In view of this, the expression o f ACE2 protein was further verified by the immunohistochemistry of ACE2 pr otein in the HPA Database (figure 2B). The results further proved that the exp ression of ACE2 protein in kidney tissue was significantly higher than that in lung tissue, and mainly expressed in renal tubular epithelial cells. 

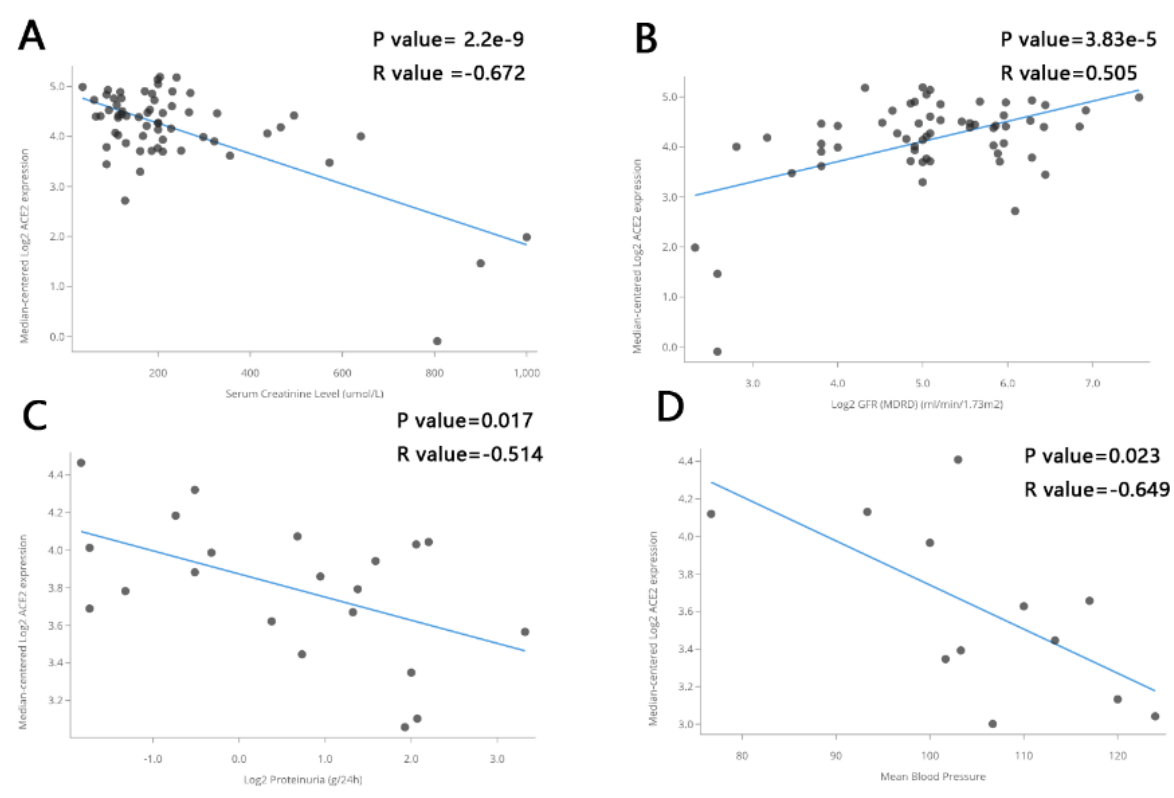

Fig.3 The relationship between the expression of ACE2 mRNA and renal funct ion. A:The expression of ACE2 mRNA was negatively correlated with creatini ne $(\mathrm{P}=2.2 \mathrm{e}-9, \mathrm{R}=-0.672, \mathrm{GSM} 240814, \mathrm{n}=66)$. B.ACE2 mRNA was positively correl ated with glomerular filtration rate $(\mathrm{p}=3.83 \mathrm{e}-5, \mathrm{r}=0.505, \mathrm{GSM} 240828, \mathrm{n}=66)$.C. ACE 2 mRNA was negatively correlated with albuminuria $(\mathrm{p}=0.017 \mathrm{r}=-0.514)$.D.ACE2 mRNA was negatively correlated with mean blood pressure $(\mathrm{P}=0.023, \mathrm{r}=-0.649)$.

(Nephreseq Database)

\section{The relationship between the expression of ACE2 mRNA and renal functio} n.

A total of 66 samples were enrolled in the study, and it was found that the e xpression of ACE2 mRNA was negatively correlated with serum creatinine $(p=$ 2.2e-9, $r=-0.672$, GSM240814 Fig.3A), and positively correlated with GFR ( $p=3.83$ e-5,r=0.505,GSM240828, Fig.3B). The study further confirmed the relationship between ACE2 and proteinuria. There was a negative correlation between ACE 2 and 24-hour urinary protein in patients with $\operatorname{IgA}$ glomerulonephritis. $(p=0.017$, $\mathrm{r}=-0.514, \mathrm{n}=21$, ERCB cohort). ACE2 protein may play a role in reducing pr oteinuria in glomerulonephritis. Previous studies have shown that ACE2 protein can negatively regulate the RAS system, which was further verified by the $\mathrm{Ne}$ phroseq Database. It was noted that in patients with diabetes, ACE2 was negat ively correlated with average blood pressure $(p=0.023, r=-0.649, n=12$, ERCB cohor t,Fig.3D). Through the study, it can be seen that ACE2 protein play an import ant protective role in kidney tissue.

\section{Complications caused by COVID-19 was higher in ARDS than in AKI.}

Clinical data, which ARDS and AKI caused by COVID-19, was collected, by using online preprinting web sites for "Pubmed Database", "BioRxiv" and "med Rxiv".Studies have demonstrated that the number of ARDS complications in pa tients with COVID-19 is much higher than that of AKI (Table 1), and ARDS is the main complication of COVID-19. We further collected data on deaths ca 
used by AKI or ARDS in patients with COVID-19. It can be observed that th e number of deaths caused by ARDS is also much higher than that caused by AKI. (Table2)

Table1 Numbers of ARDS or AKI complications in patients with COVID-19

\begin{tabular}{cccc}
\hline Number & ARDS & AKI & Source or Journal \\
\hline 41 & $12(29 \%)$ & $3(7 \%)$ & Lancet $^{[4]}$ \\
138 & $27(19.6 \%)$ & $5(3.6 \%)$ & JAMA $^{[5]}$ \\
99 & $17(17 \%)$ & $3(3 \%)$ & Lancet $^{[7]}$ \\
267 & $33(12.4 \%)$ & NA & medRxiv $^{[6]}$ \\
116 & $16(13.8 \%)$ & 0 & medRxiv $^{[17]}$ \\
710 & NA & $22(3.2 \%)$ & medRxiv $^{[18]}$ \\
109 & $53(48.6 \%)$ & NA & medRxiv $^{[19]}$ \\
\hline
\end{tabular}

Table 2 Deaths caused by AKI or ARDS in patients with COVID-19

\begin{tabular}{cccc}
\hline Death Number & ARDS & AKI & Source or Journal \\
\hline 82 & $57(69.5 \%)$ & $3(3.7 \%)$ & medRxiv $^{[20]}$ \\
25 & $25(100 \%)$ & $12(48 \%)$ & medRxiv $^{[21]}$ \\
36 & $36(100 \%)$ & $1(2.78 \%)$ & medRxiv $^{[22]}$ \\
\hline
\end{tabular}

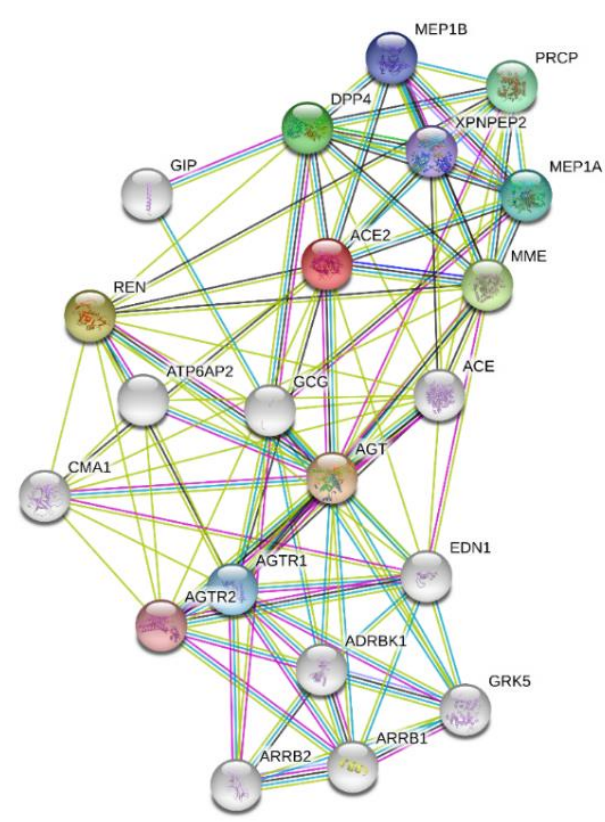

Fig.4 ACE2 protein-protein interaction (PPI) functional Network

\section{ACE2 protein-protein interaction (PPI) functional Network}

The ACE2 protein-protein interaction (PPI) was analyzed by the STRING Database. 21 
protein was shown in the PPI Network (Fig. 4). The network consists of 21 nodes and 92 edges, with an average node degree of 9.24. Links in different colors represent different meanings of biological information. Besides, 21 genes are graphically represented by network interaction, clearly describing the key positions of proteins closely related to ACE2 proteins in the PPI network. (MEP1B, PRCP, DPP4, XPNPEP2, MEP1A, GIP, ACE2, MME, REN, ACE, ATP6AP2, GCG, AGT, CMA1, EDN1, AGTR1, ADRBK1, AGTR2, GRK5, ARRB2, ARRB1)

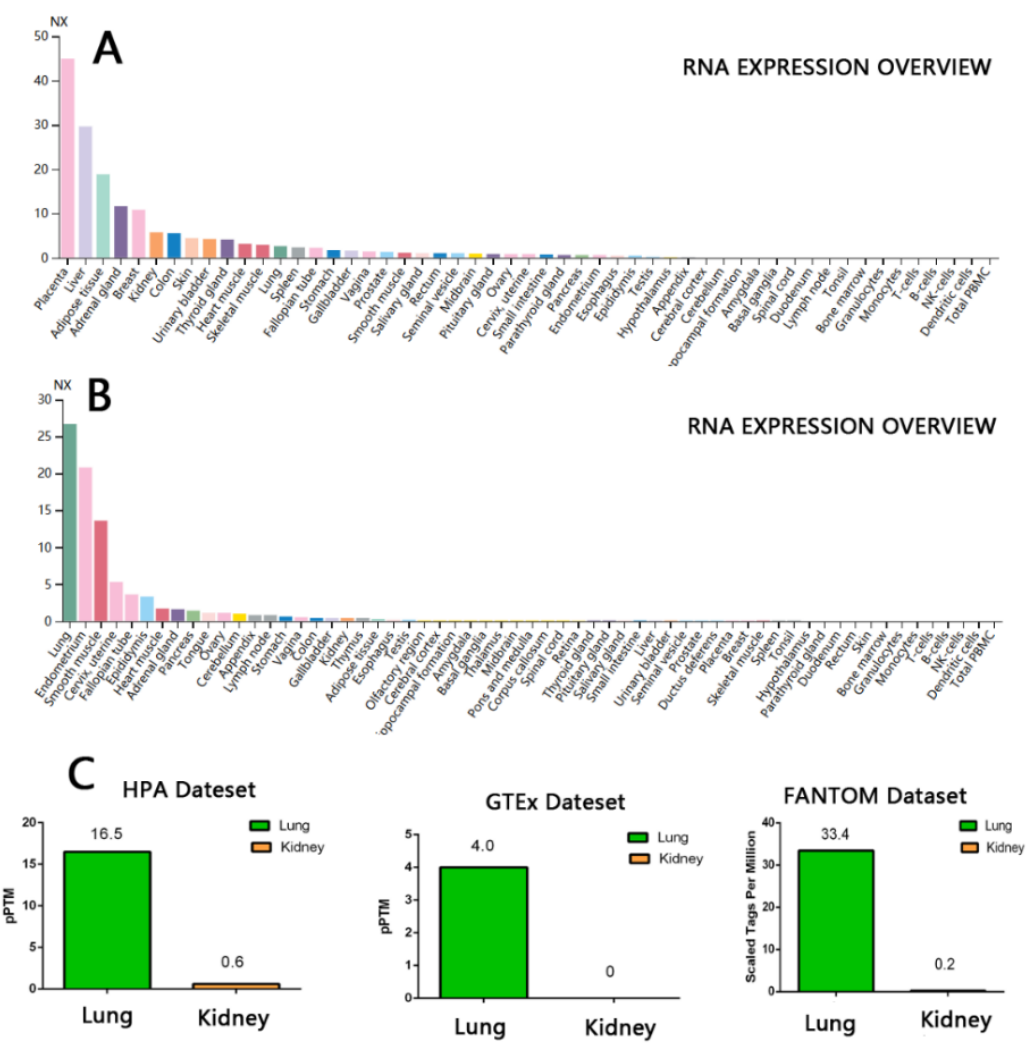

Fig.5 A: Expression of AGTR1 mRNA in organization (HPA Database).B:Expression of AGTR2 mRNA in each tissue (HPA Database).C:Expression of AGTR2 mRNA in lung and kidney. (HPA Database, GTEx Database, FANTOM5 Database)

The expression level of AGTR2 mRNA in lung tissue is higher than that in kidney tissue, and has tissue specificity.

Through STRING analysis, the 20 genes were searched by HPA, GTEx Database and FANTOM5 Database to find the genes specifically expressed in hypothetical lung tissue. Studies founded that AGTR1 is not specifically in lung tissue (Fig. 5A), but the expression of AGTR2 mRNA is very higher in lung tissue (Fig. 5B). The expression of AGTR2 mRNA was highly expressed in lung tissue, endometrium and vascular smooth muscle (Fig. 5B), while was lower in kidney tissue. In this regard, we once again get to make a systematic comparison and analysis of kidney tissue and lung tissue. The expression of AGTR2 mRNA was 16.5pPTM in lung tissue and 0.6pPTM in renal tissue. (figure 5C HPA Dataset), further proved by using the GTEx Database and FANTOM5 Database (figure 5C). From this, we infer that AGTR2 may be a potential gene for the 
different incidence of ARDS and AKI (Fig.6).

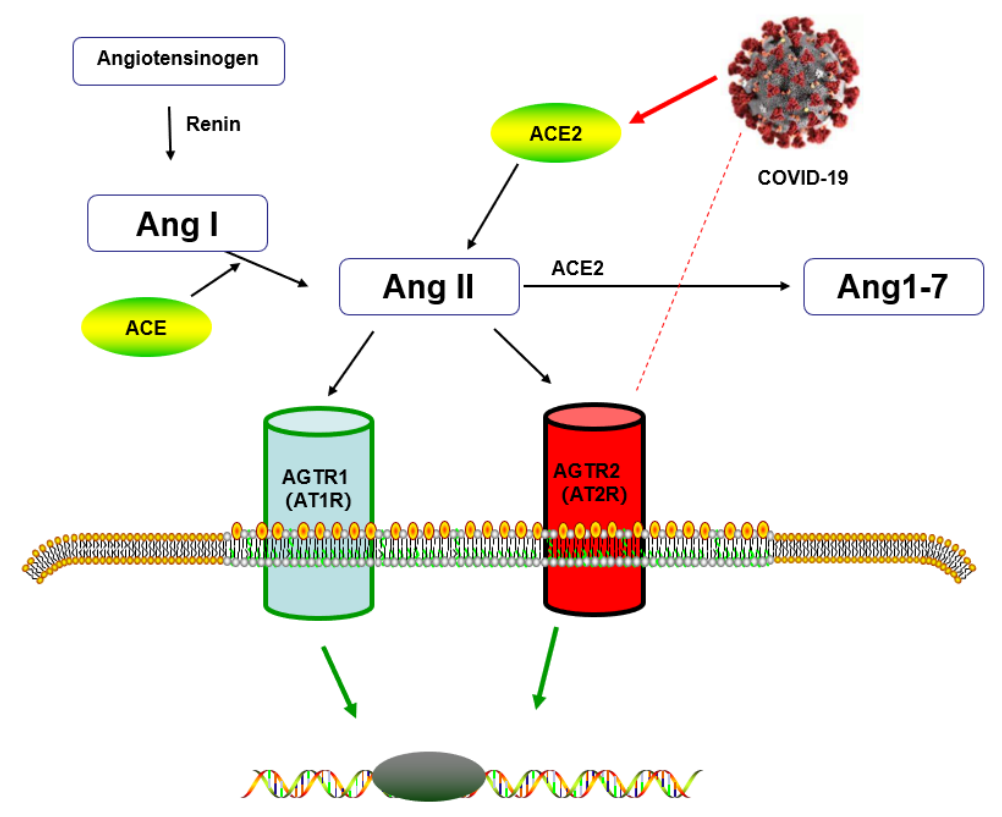

Fig. 6 Schematic diagram of lung injury model mediated by COVID-19 through AGTR2.

\section{Discussion}

COVID-19 has a three-dimensional structure of S protein that closely binds to the human cell receptor $\mathrm{ACE} 2^{[14]}$.Some studies have found that RAS inhibitors can effectively relieve acute severe pneumonia and respiratory failure ${ }^{[23]}$, so it is best to use RAS inhibitors. However, some studies show that RAS inhibitors can increase the expression of ACE2 and increase the risk of COVID-19 ${ }^{[24]}$, so it is best to stop using RAS inhibitors. Therefore, RAS inhibitors and ACE2 in the ACE/Ang-II/AT1R axis and ACE2/Ang- (1-7) / axis has become the focus of research. However, the potential mechanism and role of AGTR2 protein in the RAS system are not taken into account.

AGTR2 (angiotensin II receptor type 2) is a G protein-coupled receptor. In 2017, the structural basis of the selectivity and functional diversity of different subtypes of AGTR2 ligands will systematically be elucidated ${ }^{[25]}$. AGTR2 is generally induced by the activation of ACE2/Ang- (1-7) / MasR axis. Recent studies have revealed that Ang(1-7) may also activate AGTR2 to antagonize AGTR1 mediated effects ${ }^{[26]}$.

Built on the published literature, we summarized the data of ARDS and AKI caused by COVID-19 and found that the number of ARDS caused by COVID-19 far exceeded that of AKI. According to the distribution of ACE2 in tissues and organs, some studies have proposed that testis and kidneys are potentially damaged organs of COVID$19^{[27]}$.In the clinical study of renal injury in patients with COVID-19, the risk of death in patients with COID-19 renal injury is higher, but the complication rate of COVID19 in this study is only $3.2^{[18]}$, which is also much lower than the previously reported proportion of ARDS. However, in Wang Luwen's study ${ }^{[17]}$, a total of 116 patients with COVID-19 were enrolled in the study, and it was found that there were no clinical manifestations of acute renal injury. We can note that the incidence of AKI caused by COVID-19 is relatively low, which further indicates that there are other potential targets 
involved in COVID-19-induced lung injury.

Our study revealed that the expression of AGTR2mRNA is less in kidney tissue and the highest in lung tissue, which is tissue-specific. And in 2015 the GWAS study confirmed that the variation of the locus containing AGTR2 on the X chromosome was significantly associated with lung function in patients with cystic fibrosis ${ }^{[28]}$.In vitro, studies have demonstrated that the deficiency of the AGTR2 gene can normalize the pulmonary function index of mice with cystic pulmonary fibrosis ${ }^{[2]}$. Overexpression of the AGTR2 receptor can increase pulmonary vascular permeability and worsen pulmonary inflammation in mice with LPS-induced acute lung injury ${ }^{[30]}$. AGTR2 can also inhibit the growth of vascular smooth muscle by mediating inflammation and play an important role in the non-obstructive inflammatory injury ${ }^{[31]}$. It can be seen that AGTR2 play an important role in the occurrence and development of pulmonary fibrosis.

In this study, for the first time, the expression of ACE2 in kidney tissue was higher than that in lung tissue. Through the collection of clinical data, the lung tissue with minimal expression of ACE2 was seriously injured, and the renal tissue damage was relatively mild. Further studies found that AGTR2 may be involved in the occurrence and development of diseases caused by COVID-19. Therefore, this study reveals that AGTR2 may be a key factor in COVID-19 pneumonia. However, this study relies on the statistical analysis of the Database, and the collection of quantifiable data is based on published literature, we need to consider the limitations and shortcomings.

\section{Conclusion}

Our findings suggest that AGTR2 may be a critical factor in COVID-19 pneumonia. AGTR2 may become a potential new therapeutic target for the treatment of COVID-19 patients. Therefore, we suggest that we need in order to further explore the relationship and role of AGTR2 and COVID-19. 


\section{Reference}

[1]Wu F, Zhao S, Yu B, et al. A new coronavirus associated with human respiratory disease in China. Nature. 2020 Feb 03 doi:10.1038/s41586-020-2008-3

[2]Phelan AL, Katz R, Gostin LO. The Novel Coronavirus Originating in Wuhan, China: Challenges for Global Health Governance. JAMA. 2020. doi:10.1001/jama.2020.1097

[3]Liu J, Zheng X, Tong Q, et al. Overlapping and discrete aspects of the pathology and pathogenesis of the emerging human pathogenic coronaviruses SARS-COV, MERS-CoV, and 2019-nCoV. Journal of Medical Virology. 2020. doi:10.1002/jmv.25709

[4]Huang C, Wang Y, Li X, et al. Clinical features of patients infected with 2019 novel coronavirus in Wuhan, China. The Lancet. 2020;395: 497-506. doi:10.1016/s0140-6736(20)30183-5

[5]Wang D, Hu B, Hu C, et al. Clinical Characteristics of 138 Hospitalized Patients With 2019 Novel Coronavirus-Infected Pneumonia in Wuhan, China. JAMA. 2020. doi:10.1001/jama.2020.1585

[6]Qi D, Yan X, Tang X, et al. Epidemiological and clinical features of 2019-nCoV acute respiratory disease cases in Chongqing municipality, China: a retrospective, descriptive, multiple-center study. medRxiv. 2020.03.01. doi:10.1101/2020.03.01.20029397

[7]Chen N, Zhou M, Dong X, et al. Epidemiological and clinical characteristics of 99 cases of 2019 novel coronavirus pneumonia in Wuhan, China: a descriptive study. The Lancet. 2020;395: 507 513. doi:10.1016/s0140-6736(20)30211-7

[8]Santos RAS, Sampaio WO, Alzamora AC, et al. The ACE2/Angiotensin-(1-7)/MAS Axis of the Renin-Angiotensin System: Focus on Angiotensin-(1-7). Physiol Rev. 2018;98: 505-553. doi:10.1152/physrev.00023.2016

[9]Wösten-van Asperen RM, Lutter R, Specht PA, et al. Acute respiratory distress syndrome leads to reduced ratio of ACE/ACE2 activities and is prevented by angiotensin-(1-7) or an angiotensin II receptor antagonist. The Journal of Pathology. 2011;225: 618-627. doi:10.1002/path.2987

[10]Ge X-Y, Li J-L, Yang X-L, et al. Isolation and characterization of a bat SARS-like coronavirus that uses the ACE2 receptor. Nature. 2013;503: 535-538. doi:10.1038/nature12711

[11]Lu R, Zhao X, Li J, et al. Genomic characterisation and epidemiology of 2019 novel coronavirus: implications for virus origins and receptor binding. The Lancet. 2020;395: 565-574. doi:10.1016/s0140-6736(20)30251-8

[12]Chen Y, Guo Y, Pan Y, Zhao Z]. Structure analysis of the receptor binding of 2019-nCoV. Biochem Biophys Res Commun. 2020. doi:10.1016/j.bbrc.2020.02.071

[13]Liu Z, Xiao X, Wei X, et al. Composition and divergence of coronavirus spike proteins and host ACE2 receptors predict potential intermediate hosts of SARS-CoV-2. Journal of Medical Virology. 2020. doi:10.1002/jmv.25726

[14]Wan Y, Shang J, Graham R, Baric RS, Li F. Receptor recognition by novel coronavirus from Wuhan: An analysis based on decade-long structural studies of SARS. Journal of Virology. 2020. doi:10.1128/jvi.00127-20

[15]Chai X, Hu L, Zhang Y, et al. Specific ACE2 Expression in Cholangiocytes May Cause Liver Damage After 2019-nCoV Infection. bioRxiv preprint. 2020.02.12. doi:10.1101/2020.02.03.931766 [16]Gao QY, Chen YX, Fang JY. 2019 novel coronavirus infection and gastrointestinal tract. J Dig Dis. 2020. doi:10.1111/1751-2980.12851

[17]Wang L, Li X, Chen H, et al. SARS-CoV-2 infection does not significantly cause acute renal injury: an analysis of 116 hospitalized patients with COVID-19 in a single hospital, Wuhan, China. medRxiv. 2020.02.19. doi:10.1101/2020.02.19.20025288 
[18]Cheng $\mathrm{Y}$, Luo R, Wang K, et al. Kidney impairment is associated with in-hospital death of COVID-19 patients. medRxiv. 2020.02.18. doi:10.1101/2020.02.18.20023242

[19]Liu Y, Sun W, Li J, et al. Clinical features and progression of acute respiratory distress syndrome in coronavirus disease 2019. medRxiv. 2020.02.17. doi:10.1101/2020.02.17.20024166

[20]Zhang B, Zhou X, Qiu Y, et al. Clinical characteristics of 82 death cases with COVID-19. medRxiv. 2020.02.26. doi:10.1101/2020.02.26.20028191

[21]Li X, Wang L, Yan S, et al. Clinical characteristics of 25 death cases infected with COVID-19 pneumonia: a retrospective review of medical records in a single medical center, Wuhan, China. medRxiv . 2020.03.01. doi:10.1101/2020.02.19.20025239

[22]Huang Y, Yang R, Xu Y, Gong P. Clinical characteristics of 36 non-survivors with COVID-19 in Wuhan, China. medRxiv. 2020.02.27. doi:10.1101/2020.02.27.20029009

[23]Kuba K, Imai Y, Ohto-Nakanishi T, Penninger JM. Trilogy of ACE2: a peptidase in the reninangiotensin system, a SARS receptor, and a partner for amino acid transporters. Pharmacol Ther. 2010;128: 119-128. doi:10.1016/j.pharmthera.2010.06.003

[24]Wang P-H, Cheng Y. Increasing Host Cellular Receptor-Angiotensin-Converting Enzyme 2 (ACE2) Expression by Coronavirus may Facilitate 2019-nCoV Infection. bioRxiv preprint. 2020.February 27. doi:10.1101/2020.02.24.963348

[25]Zhang H, Han GW, Batyuk A, et al. Structural basis for selectivity and diversity in angiotensin II receptors. Nature. 2017;544: 327-332. doi:10.1038/nature22035

[26]Patel SN, Ali Q, Samuel P, Steckelings UM, Hussain T. Angiotensin II Type 2 Receptor and Receptor Mas Are Colocalized and Functionally Interdependent in Obese Zucker Rat Kidney. Hypertension. 2017;70: 831-838. doi:10.1161/HYPERTENSIONAHA.117.09679

[27]Fan C, Li K, Ding Y, Lu WL, Wang J. ACE2 Expression in Kidney and Testis May Cause Kidney and Testis Damage After 2019-nCoV Infection. medRxiv. 2020.02.12. doi:10.1101/2020.02.12.20022418

[28]Corvol H, Blackman SM, Boelle PY, et al. Genome-wide association meta-analysis identifies five modifier loci of lung disease severity in cystic fibrosis. Nat Commun. 2015;6: 8382. doi:10.1038/ncomms9382

[29]Darrah RJ, Jacono FJ, Joshi N, et al. AGTR2 absence or antagonism prevents cystic fibrosis pulmonary manifestations. J Cyst Fibros. 2019;18: 127-134. doi:10.1016/j.jcf.2018.05.013

[30]Xu X-P, Huang L-L, Hu S-L, et al. Genetic Modification of Mesenchymal Stem Cells Overexpressing Angiotensin II Type 2 Receptor Increases Cell Migration to Injured Lung in LPSInduced Acute Lung Injury Mice. STEM CELLS Translational Medicine. 2018;7: 721-730. doi:10.1002/sctm.17-0279

[31]Akishita MH, M ; Yamada, H ; Dzau, VJ. Inflammation influences vascular remodeling through AT2 receptor expression and signaling. Physiol Genomics. 2000 Jan 24 ;2(1) :13-20: 1320 